\title{
Temperature Dependence of Impurity Distributions in Nanodiamonds as Revealed by Coordinated UHV-STEM EDX and EELS Analysis
}

\author{
Rhonda Stroud and Bethany Hudak \\ US Naval Research Laboratory, Washington, District of Columbia, United States
}

Nanodiamond was the first phase identified in meteorites as having an origin older than the solar system, but this identification remains controversial [1,2]. The evidence for the presolar origin of the nanodiamonds comes from UHV stepped pyrolysis measurements of the composition of gasses released by nanodiamond isolates at temperatures of $300^{\circ} \mathrm{C}$ to $1600^{\circ} \mathrm{C}$. However, the isolates contain additional nanoscale phases, including $\mathrm{sp}^{2}$ carbon, $\mathrm{SiC}$ and refractory metal nuggets, and the stepped pyrolysis experiments do not distinguish gasses released from the different materials. The partitioning of the impurities that provide the evidence of presolar origin among the diamonds and other phases is unknown, and the majority of the diamonds may have a solar system origin. Heating stage experiments in a UHV scanning transmission electron microscope can address this information gap, by enabling direct observation of the evolution of the distribution of impurity atoms in the nanodiamonds and associated phases as a function of temperature.

To carry out temperature-dependent studies of the impurity distributions in meteoritic nanodiamond isolates, we use a Protochips Fusion heating holder in a Nion UltraSTEM200-X. Our UltraSTEM200-X is equipped with a Gatan Enfinium ER electron energy loss spectrometer (EELS) and a 0.7 sr Bruker Xflash energy dispersive x-ray (EDX) spectrometer. In order to permit coordinated EELS and EDX measurements, we modified the Fusion holder to mitigate shadowing of x-rays emitted from the sample. The nanodiamond samples are prepared by pipetting aliquots of the isolates onto holey carbon membrane heating chips. Heating was performed in $50^{\circ} \mathrm{C}$ steps from room temperature to $1200^{\circ} \mathrm{C}$, the manufacturer's recommended temperature limit for the heating chips. The transformation of the nanodiamonds and associated phases was tracked with high angle and medium angle dark field images, and C-K edge EELS and EDX measurements.

Initial measurements (Fig. 1) were performed with an unmodified Fusion holder. The as-deposited sample showed the characteristic C-K edge EEL fine-structure of diamond, along with a small p* peak at 284.3 $\mathrm{eV}$, indicative of the $\mathrm{sp}^{2}$ component common to nanodiamonds. In addition, there was a small peak at $\sim 282.4 \mathrm{eV}$, reported to be associated with $\mathrm{N}$ impurities in diamond ( $\mathrm{N}-\mathrm{V}$ center) [3]. The intensity of $\mathrm{N}$ defect peak was constant until $\sim 400^{\circ} \mathrm{C}$, and then decreased significantly between $400^{\circ} \mathrm{C}$ and $600^{\circ} \mathrm{C}$, and was no longer detectable at $700^{\circ} \mathrm{C}$, which correspond to the temperature release range for the predominant solar system gas component in stepped pyrolysis experiments. Further heating to $1200^{\circ} \mathrm{C}$ resulted in a transformation to at least $50 \%$ graphitic carbon, and loss of up to $50 \%$ of the sample mass.

Additional measurements with coordinated EDX and EELS using the modified holder are planned. Initial tests at room temperature verified that the EDX signal from the sample could be obtained, although system peaks in $\mathrm{C}, \mathrm{Si}, \mathrm{Al}$ and $\mathrm{Cu}$ were also observed. No system peaks in $\mathrm{N}$ were detected, which indicates that tracking of the total $\mathrm{N}$ content of the individual $2 \mathrm{~nm}$ diamond particles with EDX [4] and the N-V abundance with C-K edge EELS will be possible [5]. 

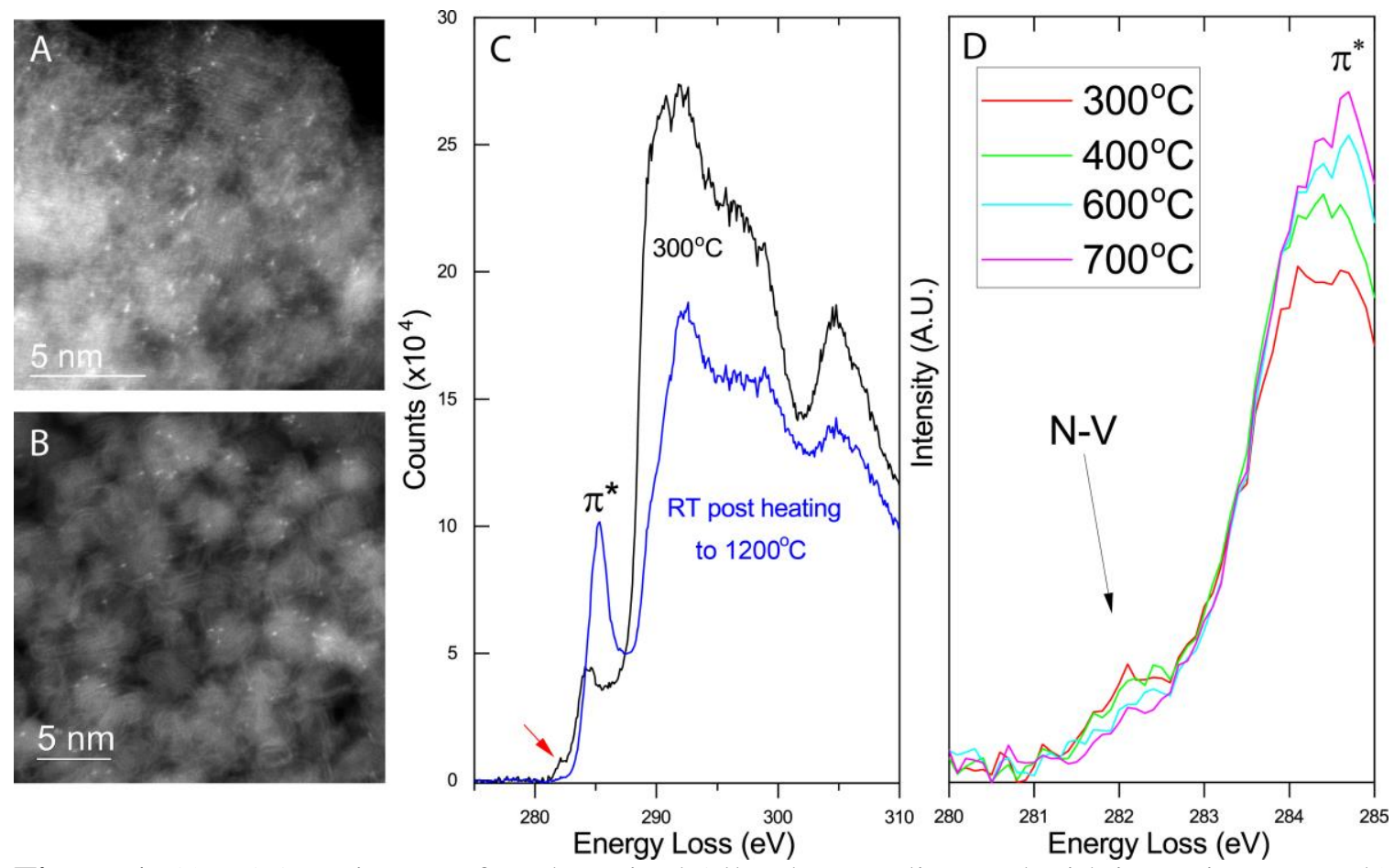

Figure 1. A) HAADF image of as-deposited Allende nanodiamond with impurity atoms. b) Nanodiamond partially transformed to graphitic carbon after heating to $1200^{\circ} \mathrm{C}$. C) Comparison of C-K edge electron energy dispersive loss spectra before and after heating. D) Decrease of the $\mathrm{N}-\mathrm{V}$ center feature on heating from $300^{\circ} \mathrm{C}$ to $700^{\circ} \mathrm{C}$.

\section{References}

[1] R. Lewis et al. Nature 326 (1987), p.162.

[2] U. Ott et al. Publications of the Astronomical Society of Australia 29 (2012), p. 90.

[3] S. L. Y. Chang et al. Nanoscale 8 (2016), p. 10548.

[4] R. M. Stroud et al. Applied Phys. Lett. 108 (2016), p. 163101.

[5] The authors acknowledge funding from the NASA Emerging Worlds Program, grant 80HQTR19T0038. J. Greer and P. Heck, and C. Alexander are gratefully acknowledged for providing Allende and Murchison nanodiamond isolates, respectively. 